\title{
A Reliable and Useful Method to Determine the Saturation State from Helmholtz Energy Equations of State*
}

\author{
Ryo AKASAKA** \\ ** Faculty of Humanities, Kyushu Lutheran College \\ 3-12-16 Kurokami, Kumamoto 860-8520, Japan \\ E-mail: akasaka@klc.ac.jp
}

\begin{abstract}
This paper discusses iterative calculation methods to determine the saturation state of pure fluids by using Helmholtz energy equations of state. The commonly used method is based on the successive substitution approach. This method is simple, but requires an aid of ancillary equations to estimate sufficiently accurate initial guesses for the saturation pressure and saturated liquid and vapor densities. In particular, in the vicinity of the critical point very accurate initial guesses are needed. This paper presents an alternative method based on the Newton-Raphson approach. The alternative method does not require accurate initial guesses. Therefore, the aid of ancillary equations is unnecessary. The iterative process is more stable than that in the commonly used method, even if rough initial guesses are given. The usability of the alternative method is demonstrated for several published equations of state.
\end{abstract}

Key words : Saturation Pressure, Saturated Liquid Density, Saturated Vapor Density, Helmholtz Energy Equation of State, Numerical Calculation

\section{Introduction}

Modern equations of state are often formulated using the Helmholtz free energy as the fundamental property with independent variables of temperature and density. For the last two decades, highly accurate fundamental equations of state explicit in the Helmholtz free energy have been developed for many industrial fluids such as methane ${ }^{(1)}$, nitrogen ${ }^{(2)}$, carbon dioxide $^{(3)}$, and water ${ }^{(4)}$. Software for calculating thermodynamic properties of fluids using these fundamental equations of state, such as REFPROP ${ }^{(5)}$ and PROPATH ${ }^{(6)}$, is now available.

While all thermodynamic properties of a single phase can be calculated from the fundamental equations of state either directly or by differentiation of the equations, the saturation state is determined from the Maxwell criterion. In general, iterative calculations are needed to solve the criterion using Helmholtz energy equations of state. The most extensively used approach for the calculations is the successive substitution method. The method is simple and works well if sufficiently accurate initial guesses for the saturation pressure and the saturated liquid and vapor densities are given. Therefore, an aid of ancillary equations to estimate accurate initial guesses is essential. In particular, in the vicinity of the critical point the method requires very accurate initial guesses. An absence of such equations makes the method troublesome. Although the feature of the method was investigated in detail ${ }^{(7)}$, an alternative method to overcome the shortcomings has not been proposed yet.

This paper presents a more reliable method for determining the saturation state from Helmholtz energy equations of state. No ancillary equation to estimate initial guesses is used in the method, because the method is much less sensitive to the initial guess accuracies. Additionally, the method uses a single algorithm for the temperatures from the triple point to the critical point. Modifications for the temperatures near the critical point are unnecessary. 
To show the usability, applications of the method to several published equations of state are demonstrated. The method provides a very useful technique for computer programming.

\section{Nomenclature}

$\underline{A}:$ molar Helmholtz free energy, $\mathrm{J} / \mathrm{mol}$

$\underline{G}$ : molar Gibbs free energy, $\mathrm{J} / \mathrm{mol}$

$p$ : pressure, $\mathrm{Pa}$

$p_{s}:$ saturation pressure, $\mathrm{Pa}$

$R_{m}$ : universal gas constant, $\mathrm{J} / \mathrm{mol} \cdot \mathrm{K}$

$\underline{S}:$ molar entropy, $\mathrm{J} / \mathrm{mol} \cdot \mathrm{K}$

$T$ : temperature, $\mathrm{K}$

$\underline{U}$ : molar internal energy, $\mathrm{J} / \mathrm{mol}$

$\alpha$ : dimensionless Helmholtz free energy

$\gamma:$ parameter to slow the convergence speed

$\delta:$ reduced density

$\rho:$ density

$\rho^{\prime}$ : staturated liquid density

$\rho^{\prime \prime}$ : staturated vapor density

$\tau$ : inverse reduced temperature

\section{Superscripts}

$\circ$ : ideal-gas contribution

$r$ : residual part

\section{Subscripts}

$c:$ critical point

$t r$ : triple point

\section{Calculation method}

For pure fluids, the equations of state explicit in the Helmholtz free energy usually has the form

$$
\frac{\underline{A}}{R_{m} T}=\alpha(\tau, \delta)=\alpha^{\circ}(\tau, \delta)+\alpha^{r}(\tau, \delta),
$$

where $\underline{A}=\underline{U}-T \underline{S}$ is the molar Helmholtz free energy, $R_{m}$ is the universal gas constant, $\alpha$ is the dimensionless Helmholtz free energy, $\alpha^{\circ}$ is the ideal-gas contribution to $\alpha$, and $\alpha^{r}$ is the residual part of $\alpha$. Independent variables in Eq. (1) are the inverse reduced temperature $\tau$ and the reduced density $\delta$, which are defined as

$$
\tau=\frac{T_{c}}{T}
$$

and

$$
\delta=\frac{\rho}{\rho_{c}} .
$$

All thermodynamic properties of a single phase can be calculated from the fundamental equation of state given in Eq. (1). For example, the pressure $p$ and the molar Gibbs free energy $\underline{G}$ are

$$
\frac{p}{R_{m} T \rho}=1+\delta \alpha_{\delta}^{r}
$$

and

$$
\frac{\underline{G}}{R_{m} T}=1+\alpha^{\circ}+\alpha^{r}+\delta \alpha_{\delta}^{r}
$$


where $\alpha_{\delta}^{r}$ is the first-order partial derivative of $\alpha^{r}$ with respect to $\delta$, which is defined by

$$
\alpha_{\delta}^{r}(\tau, \delta)=\left(\frac{\partial \alpha^{r}}{\partial \delta}\right)_{\tau} .
$$

According to the Maxwell criterion, the equilibrium of the liquid phase (') and vapor phase (") can be determined by the conditions

$$
\begin{aligned}
& p_{s}=p\left(\tau, \delta^{\prime}\right)=R_{m} T \rho^{\prime}\left[1+\delta \alpha_{\delta}^{r}\left(\tau, \delta^{\prime}\right)\right], \\
& p_{s}=p\left(\tau, \delta^{\prime \prime}\right)=R_{m} T \rho^{\prime \prime}\left[1+\delta \alpha_{\delta}^{r}\left(\tau, \delta^{\prime \prime}\right)\right],
\end{aligned}
$$

and

$$
\underline{G}=\underline{G}^{\prime}
$$

where $\delta^{\prime}=\rho^{\prime} / \rho_{c}, \delta^{\prime \prime}=\rho^{\prime \prime} / \rho_{c}$, and $p_{s}$ is the saturation pressure. Using Eq. (5), the final condition can be rewritten as

$$
\alpha^{\circ}\left(\tau, \delta^{\prime}\right)+\alpha^{r}\left(\tau, \delta^{\prime}\right)+\delta^{\prime} \alpha_{\delta}^{r}\left(\tau, \delta^{\prime}\right)=\alpha^{\circ}\left(\tau, \delta^{\prime \prime}\right)+\alpha^{r}\left(\tau, \delta^{\prime \prime}\right)+\delta^{\prime \prime} \alpha_{\delta}^{r}\left(\tau, \delta^{\prime \prime}\right) .
$$

Generally, $\alpha^{\circ}$ is expressed in the form

$$
\alpha^{\circ}(\tau, \delta)=\ln \delta+\beta^{\circ}(\tau)
$$

where $\beta^{\circ}$ is a temperature dependent part of the ideal-gas contribution. Therefore, Eq. (10) is

$$
\ln \delta^{\prime}+\alpha^{r}\left(\tau, \delta^{\prime}\right)+\delta^{\prime} \alpha_{\delta}^{r}\left(\tau, \delta^{\prime}\right)=\ln \delta^{\prime \prime}+\alpha^{r}\left(\tau, \delta^{\prime \prime}\right)+\delta^{\prime \prime} \alpha_{\delta}^{r}\left(\tau, \delta^{\prime \prime}\right)
$$

Substituting Eqs. (7) and (8) into Eq. (12), the following equation is obtained:

$$
p_{s}=\frac{R_{m} T \rho^{\prime} \rho^{\prime \prime}}{\rho^{\prime}-\rho^{\prime \prime}}\left[\alpha^{r}\left(\tau, \delta^{\prime}\right)-\alpha^{r}\left(\tau, \delta^{\prime \prime}\right)+\ln \frac{\delta^{\prime}}{\delta^{\prime \prime}}\right] .
$$

For a given temperature, the simplest way to find the saturation pressure $p_{s}$ and the saturated liquid and vapor densities, $\rho^{\prime}$ and $\rho^{\prime \prime}$, satisfying Eqs. (7), (8), and (12) simultaneously is a successive substitution method using $p_{s}$ as the independent variable in the iteration. Figure 1 shows the flow chart for this method. Initially, an appropriate guesses for $p_{s}$ is selected $\left(p_{s 1}\right)$, and $\delta^{\prime}$ and $\delta^{\prime \prime}$ are calculated from the $p_{s 1}$ using Eqs. (7) and (8). Then, $p_{s}$ is recalculated using the $\delta^{\prime}$ and $\delta^{\prime \prime}$ according to Eq. (13) ( $\left.p_{s 2}\right)$. If the $p_{s 1}$ is equal to the $p_{s 2}$, the $p_{s 1}$ is the saturation pressure at the temperature. If not, the $p_{s 1}$ is modified. The simple successive substitution method uses the $p_{s 2}$ for the next $p_{s 1}$.

Since the convergence speed of the simple successive substitution method is generally slow, more effective algorithms have been proposed. REFPROP ${ }^{(5)}$ improves a guess for $p_{s}$ using the relation

$$
p_{s}^{(k+1)}=p_{s}^{(k)}-\frac{\underline{G}\left(T, \rho^{\prime}\right)-\underline{G}^{\prime}\left(T, \rho^{\prime \prime}\right)}{1 / \rho^{\prime}-1 / \rho^{\prime \prime}} .
$$

Span $^{(7)}$ proposed a method applying the regula falsi algorithm. This method modifies a guess for $p_{s}$ according to the arithmetic rule

$$
p_{s}^{(k+1)}=p_{s}^{(k)}-\frac{\left[p_{s}^{(k)}-p_{s}^{(k-1)}\right] R E S^{(k)}}{R E S^{(k)}-R E S^{(k-1)}},
$$

where $R E S^{(k)}$ is a residuum defined as

$$
R E S^{(k)}=\frac{R_{m} T \rho^{\prime} \rho^{\prime \prime}}{\rho^{\prime}-\rho^{\prime \prime}}\left[\alpha^{r}\left(\tau, \delta^{\prime}\right)-\alpha^{r}\left(\tau, \delta^{\prime \prime}\right)+\ln \frac{\delta^{\prime}}{\delta^{\prime \prime}}\right]-p_{s}^{(k)} .
$$

Because of the simplicity, the successive substitution method using $p_{s}$ as the independent variable in the iteration has commonly been used in many applications. However, as pointed out by $\operatorname{Span}^{(7)}$, some difficulties are inherent in implementing the method. Figure 2 shows an 


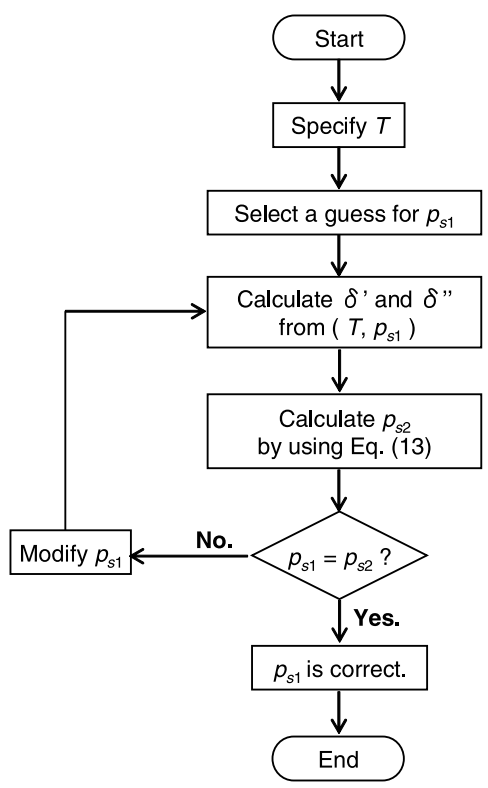

Fig. 1 Successive substitution method using $p_{s}$ as the independent variable in the iteration

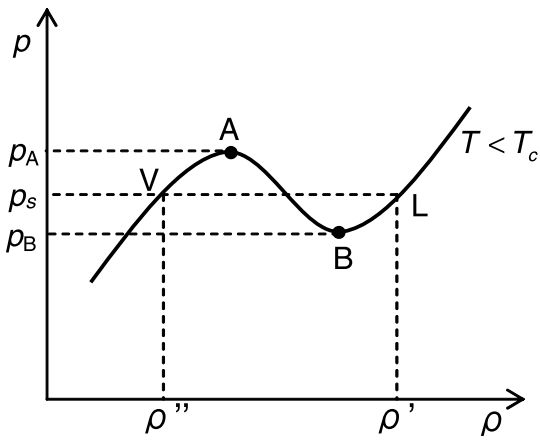

Fig. 2 An isotherm at $T<T_{c}$ on $\rho$ - $p$ diagram

isotherm at $T<T_{c}$ on $\rho-p$ diagram. The points $\mathrm{A}$ and $\mathrm{B}$ are the spinodal points, and the path V-A-B-L represents the Maxwell loop. A guess for $p_{s}$ must always be in between $p_{\mathrm{A}}$ and $p_{\mathrm{B}}$, because it is necessary to find the densities both for the liquid and vapor phase from the guess. Since at $T \simeq T_{c}$ the Maxwell loop is almost flat and the gap between $p_{\mathrm{A}}$ and $p_{\mathrm{B}}$ is narrow, special attention should be paid to select and modify the guess for $p_{s}$. For example, $\operatorname{REFPROP}^{(5)}$ gives an initial guess for $p_{s}$ by $p_{s}=\left(p_{\mathrm{A}}+p_{\mathrm{B}}\right) / 2$. However, many calculation steps may be needed to find $p_{\mathrm{A}}$ and $p_{\mathrm{B}}$.

Another difficulty arises in finding densities $\rho^{\prime}$ and $\rho^{\prime \prime}$ from a guess for $p_{s}$. Iterative calculations are certainly needed because Eqs. (7) and (8) are written in a pressure-explicit form. Accurate initial guesses for $\rho^{\prime}$ and $\rho^{\prime \prime}$ have to be given to ensure desirable convergence. Some equations of state provide ancillary equations giving sufficiently accurate guesses for $\rho^{\prime}$ and $\rho^{\prime \prime}$. If such equations are not provided, the calculation for finding $\rho^{\prime}$ and $\rho^{\prime \prime}$ becomes troublesome.

An alternative method to solve the Maxwell relation is presented here. This method is based on the Newton-Raphson algorithm for simultaneous equations. Figure 3 shows the flow chart for the method.

Eliminating $p_{s}$ from Eqs. (7), (8), and (12) yields the following simultaneous equations 


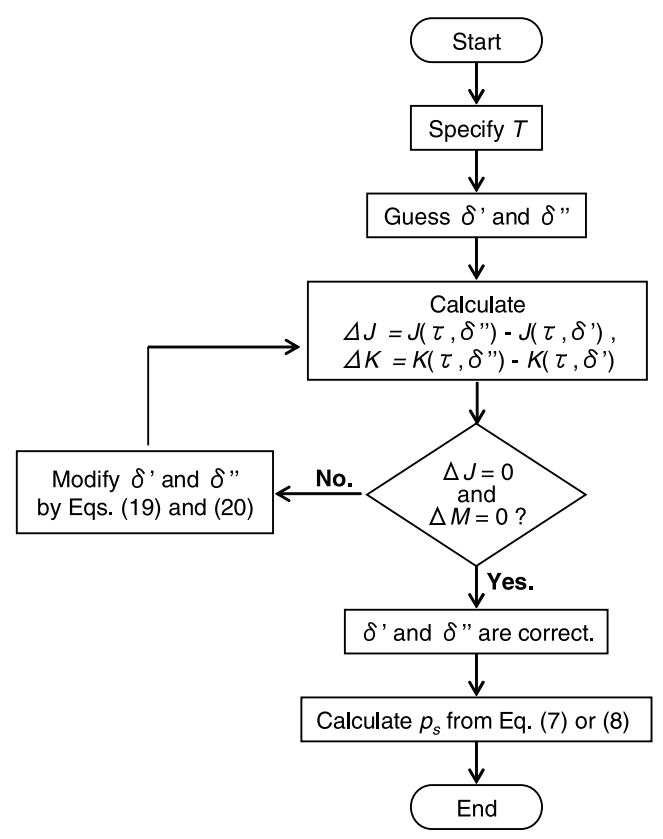

Fig. 3 Newton-Raphson method for simultaneous equations to find $\delta^{\prime}$ and $\delta^{\prime \prime}$

for $\delta^{\prime}$ and $\delta^{\prime \prime}$ :

$$
\delta^{\prime \prime}\left[1+\delta^{\prime \prime} \alpha_{\delta}^{r}\left(\tau, \delta^{\prime \prime}\right)\right]-\delta^{\prime}\left[1+\delta^{\prime} \alpha_{\delta}^{r}\left(\tau, \delta^{\prime}\right)\right]=0
$$

and

$$
\delta^{\prime \prime} \alpha_{\delta}^{r}\left(\tau, \delta^{\prime \prime}\right)+\alpha^{r}\left(\tau, \delta^{\prime \prime}\right)+\ln \delta^{\prime \prime}-\left[\delta^{\prime} \alpha_{\delta}^{r}\left(\tau, \delta^{\prime}\right)+\alpha^{r}\left(\tau, \delta^{\prime}\right)+\ln \delta^{\prime}\right]=0 .
$$

The Newton-Raphson algorithm for simultaneous equations calculates better approximations for $\delta^{\prime}$ and $\delta^{\prime \prime}$ using the equations

$$
\begin{aligned}
\delta^{(k+1)}=\delta^{\prime(k)}+\frac{\gamma}{\Delta}\{ & {\left[K\left(\tau, \delta^{\prime \prime}\right)-K\left(\tau, \delta^{\prime}\right)\right] J_{\delta}\left(\tau, \delta^{\prime \prime}\right) } \\
- & {\left.\left[J\left(\tau, \delta^{\prime \prime}\right)-J\left(\tau, \delta^{\prime}\right)\right] K_{\delta}\left(\tau, \delta^{\prime \prime}\right)\right\} }
\end{aligned}
$$

and

$$
\begin{aligned}
\delta^{\prime \prime(k+1)}=\delta^{\prime \prime(k)}+\frac{\gamma}{\Delta}\{ & {\left[K\left(\tau, \delta^{\prime \prime}\right)-K\left(\tau, \delta^{\prime}\right)\right] J_{\delta}\left(\tau, \delta^{\prime}\right) } \\
- & {\left.\left[J\left(\tau, \delta^{\prime \prime}\right)-J\left(\tau, \delta^{\prime}\right)\right] K_{\delta}\left(\tau, \delta^{\prime}\right)\right\}, }
\end{aligned}
$$

where $\gamma$ is the adjustable parameter to slow the convergence speed, and $J, K, J_{\delta}, K_{\delta}$, and $\Delta$ are defined as

$$
\begin{aligned}
& J(\tau, \delta)=\delta\left[1+\delta \alpha_{\delta}^{r}(\tau, \delta)\right], \\
& K(\tau, \delta)=\delta \alpha_{\delta}^{r}(\tau, \delta)+\alpha^{r}(\tau, \delta)+\ln \delta, \\
& J_{\delta}(\tau, \delta)=\left(\frac{\partial J}{\partial \delta}\right)_{\tau}=1+2 \delta \alpha_{\delta}^{r}+\delta^{2} \alpha_{\delta \delta}^{r}, \\
& K_{\delta}(\tau, \delta)=\left(\frac{\partial K}{\partial \delta}\right)_{\tau}=2 \alpha_{\delta}^{r}+\delta \alpha_{\delta \delta}^{r}+\frac{1}{\delta},
\end{aligned}
$$

and

$$
\Delta=J_{\delta}\left(\tau, \delta^{\prime \prime}\right) K_{\delta}\left(\tau, \delta^{\prime}\right)-J_{\delta}\left(\tau, \delta^{\prime}\right) K_{\delta}\left(\tau, \delta^{\prime \prime}\right)
$$




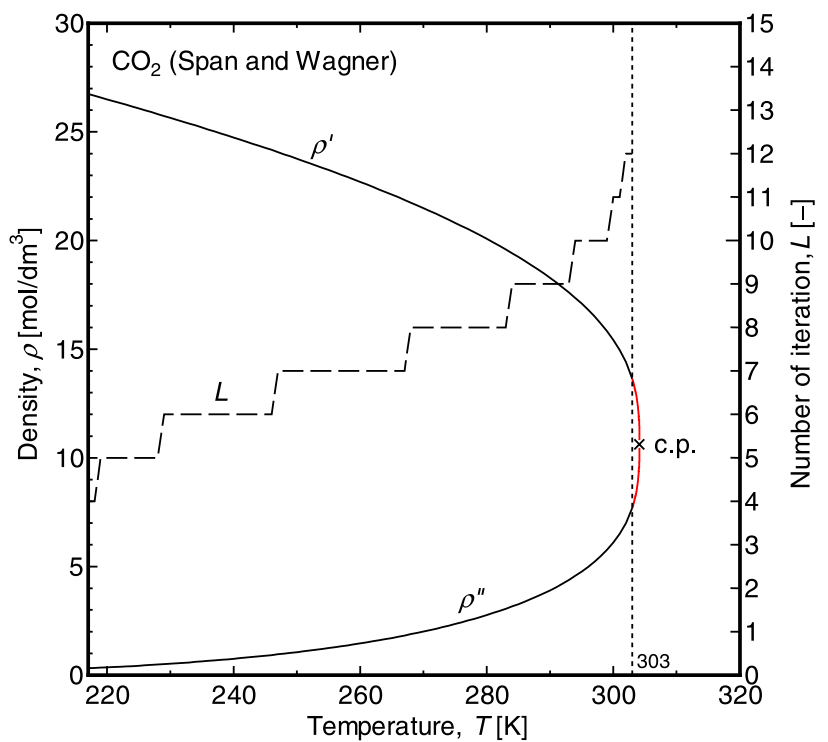

Fig. 4 Saturated liquid and vapor densities of carbon dioxide calculated from the equation of state developed by Span and Wagner ${ }^{(3)}$. The black solid lines represent results of the calculations using the saturated densities at the triple point as the initial guesses. The red solid lines denote results using the saturated densities at $303 \mathrm{~K}$ as the initial guesses.

The saturation pressure can be calculated from a root of either $\delta^{\prime}$ or $\delta^{\prime \prime}$ using Eqs. (7) or (8). The parameter $\gamma$ is normally set to unity. If the iteration with $\gamma=1$ is less successful, the value for $\gamma$ should be reduced.

Applications of the method to several equations of state revealed two requirements for initial guesses. The first requirement is that an initial guess for $\rho^{\prime}$ has to be larger than the root of $\rho^{\prime}$, and the second is that an initial guess for $\rho^{\prime \prime}$ has to be smaller than the root of $\rho^{\prime \prime}$. If these two requirements are satisfied, values for the initial guesses do not need to be accurate. Therefore, the saturated densities at the triple point, which are the largest liquid density and the smallest vapor density in the vapor-liquid coexistence region, can be used as initial guesses over a wide range of temperatures. It is recommended that another initial guesses are used near the critical point, where the saturated densities exhibit strong temperature dependence.

\section{Results and Discussion}

The presented method was applied to the equation of state for carbon dioxide developed by Span and Wagner ${ }^{(3)}$. The functional form of $\alpha^{r}$ is expressed as

$$
\begin{aligned}
\alpha^{r}(\tau, \delta)= & \sum_{i=1}^{7} a_{i} \delta^{d_{i}} \tau^{t_{i}}+\sum_{i=8}^{34} a_{i} \delta^{d_{i}} \tau^{t_{i}} \exp \left(-\delta^{e_{i}}\right) \\
& +\sum_{i=35}^{39} a_{i} \delta^{d_{i}} \tau^{t_{i}} \exp \left[-C_{i}\left(\delta-b_{i}\right)^{2}-D_{i}\left(\tau-c_{i}\right)^{2}\right]+\sum_{i=40}^{42} a_{i} \delta \Lambda_{i}^{b_{i}} \Psi_{i},
\end{aligned}
$$

where

$$
\begin{aligned}
& \Psi_{i}=\exp \left[-C_{i}(\delta-1)^{2}-D_{i}(\tau-1)^{2}\right], \\
& \Lambda_{i}=\theta_{i}^{2}+B_{i}\left[(\delta-1)^{2}\right]^{c_{i}},
\end{aligned}
$$

and

$$
\theta_{i}=(1-\tau)+A_{i}\left[(\delta-1)^{2}\right]^{1 /\left(2 \beta_{i}\right)} .
$$




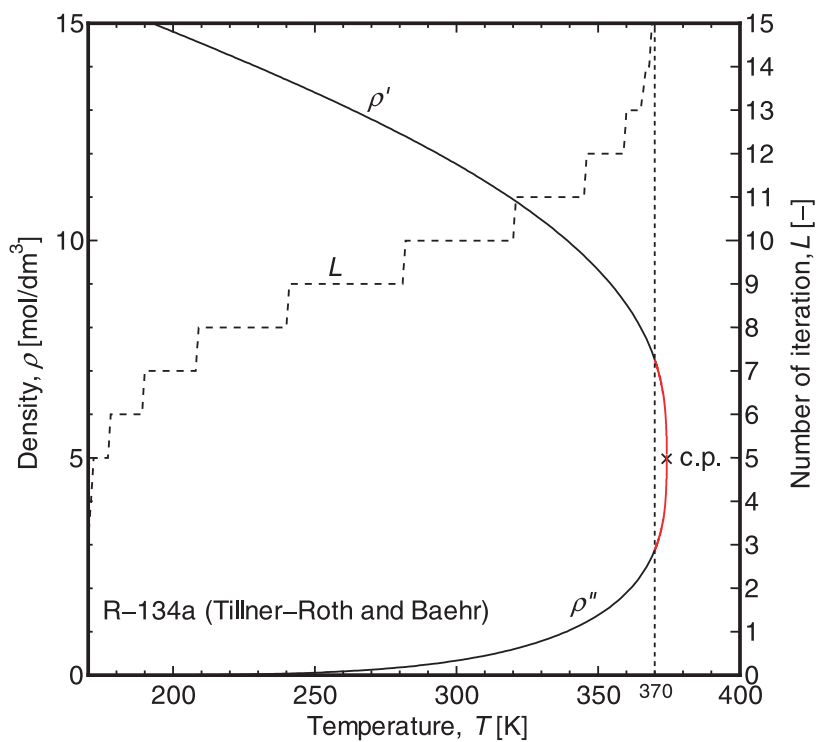

Fig. 5 Saturated liquid and vapor densities of R-134a calculated from the equation of state developed by Tillner-Roth and Baehr ${ }^{(8)}$. The black solid lines represent results of the calculations using the saturated densities at the triple point as the initial guesses. The red solid lines denote results using the saturated densities at $370 \mathrm{~K}$ as the initial guesses.

The coefficients and exponents $A_{i}, B_{i}, C_{i}, D_{i}, a_{i}, b_{i}, c_{i}, d_{i}, e_{i}, t_{i}$, and $\beta_{i}$ are presented elsewhere ${ }^{(3)}$. Span and Wagner ${ }^{(3)}$ developed the following ancillary equations together with the equation of state:

$$
\begin{aligned}
& \ln \left(\frac{p_{s}}{p_{c}}\right)=\frac{1}{\theta} \sum_{i=1}^{4} a_{i}(1-\theta)^{t_{i}}, \\
& \ln \left(\frac{\rho^{\prime}}{\rho_{c}}\right)=\sum_{i=1}^{4} a_{i}(1-\theta)^{t_{i}},
\end{aligned}
$$

and

$$
\ln \left(\frac{\rho^{\prime \prime}}{\rho_{c}}\right)=\sum_{i=1}^{5} a_{i}(1-\theta)^{t_{i}},
$$

where $\theta=T / T_{c}$. The coefficients $a_{i}$ and exponents $t_{i}$ of each equation are presented elsewhere $^{(3)}$. These equations can very accurately reproduce the saturation pressures and saturated liquid and vapor densities calculated from the equation of state. The successive substitution method always requires an aid of the equations.

The presented method can determine the saturation state without the use of the ancillary equations. The calculations of the saturated liquid and vapor densities were attempted for the temperatures from the triple point $(216.592 \mathrm{~K})$ to the critical point $(304.1282 \mathrm{~K})$. The saturated liquid and vapor densities at the triple point $\left(\rho^{\prime}=26.78 \mathrm{~mol} / \mathrm{dm}^{3}\right.$ and $\rho^{\prime \prime}=$ $0.3127 \mathrm{~mol} / \mathrm{dm}^{3}$ ) were always used as initial guesses for the presented method. The parameter $\gamma$ is set to unity. The following convergence condition was used:

$$
\left|K\left(\tau, \delta^{\prime \prime}\right)-K\left(\tau, \delta^{\prime}\right)\right|+\left|J\left(\tau, \delta^{\prime \prime}\right)-J\left(\tau, \delta^{\prime}\right)\right|<10^{-8} .
$$

Figure 4 shows results of this attempt. The number of iterations until convergence, $L$, is also plotted in the figure. It is remarkable that all calculations for the temperatures from the triple point up to $303 \mathrm{~K}$ successfully converged within 12 iterations. This upper limit ( $303 \mathrm{~K})$, which is denoted by the dotted line in Fig. 4 , is about $1.1 \mathrm{~K}$ lower than the critical point. The saturated densities at the upper limit were calculated as $\rho^{\prime}=13.63 \mathrm{~mol} / \mathrm{dm}^{3}$ and $\rho^{\prime \prime}=7.703 \mathrm{~mol} / \mathrm{dm}^{3}$, 


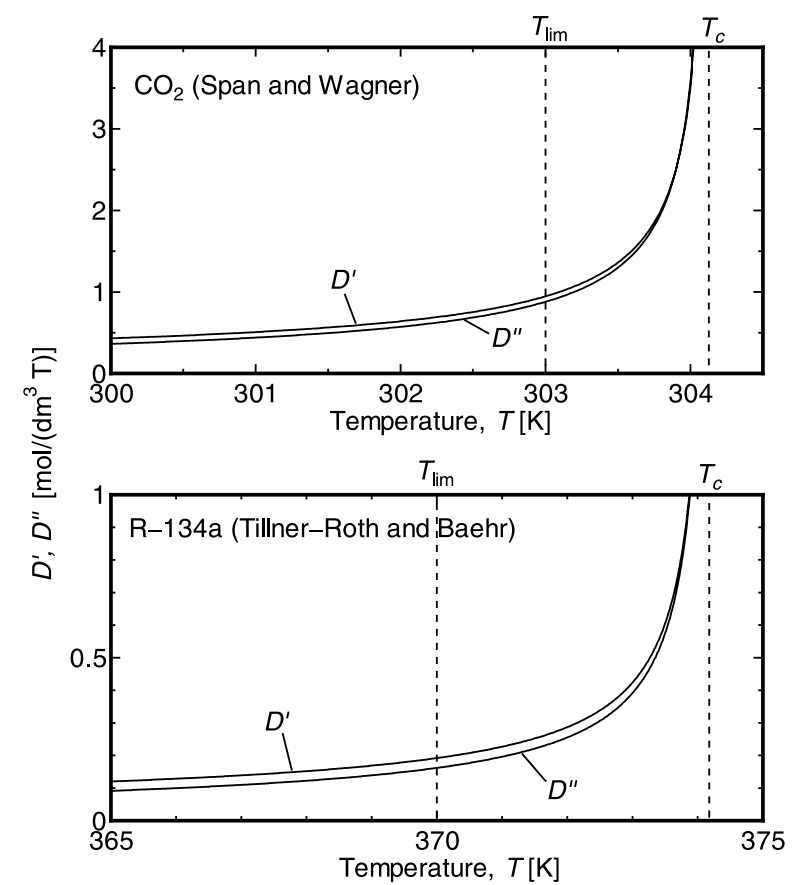

Fig. 6 Changes in the temperature dependences of the saturated liquid and vapor densities for carbon dioxide and R-134a. $D^{\prime}$ and $D^{\prime \prime}$ are defined as $D^{\prime}=$ $\left|d \rho^{\prime} / d T\right|$ and $D^{\prime \prime}=\left|d \rho^{\prime \prime} / d T\right|$.

which are greatly different from the initial guesses. By using these densities as initial guesses, the calculations from the upper limit to the critical point converged successfully.

Figure 5 shows the result of the same attempt for R-134a (1,1,1,2-Tetrafluoroethane). The triple point densities $\rho^{\prime}=15.59 \mathrm{~mol} / \mathrm{dm}^{3}$ and $\rho^{\prime \prime}=0.2761 \times 10^{-3} \mathrm{~mol} / \mathrm{dm}^{3}$ were always used as initial guesses. The calculations up to $370 \mathrm{~K}$ converged within 15 iterations. This upper limit $(370 \mathrm{~K})$ is about $4 \mathrm{~K}$ lower than the critical point $(374.18 \mathrm{~K})$. By using the densities at the upper limit $\left(\rho^{\prime}=7.256 \mathrm{~mol} / \mathrm{dm}^{3}\right.$ and $\left.\rho^{\prime \prime}=2.881 \mathrm{~mol} / \mathrm{dm}^{3}\right)$ as initial guesses, the calculations from the upper limit to the critical point converged.

In order to clarify a significance of the upper limit, $T_{\lim }$, the temperature dependences of the saturated densities defined as

$$
D^{\prime}=\left|\frac{d \rho^{\prime}}{d T}\right|
$$

and

$$
D^{\prime \prime}=\left|\frac{d \rho^{\prime \prime}}{d T}\right|
$$

were investigated. Figure 6 shows the changes in $D^{\prime}$ and $D^{\prime \prime}$ for carbon dioxide and for R134a. The locations of $T_{\lim }$ and $T_{c}$ are also shown in the figure. While $D^{\prime}$ and $D^{\prime \prime}$ increase to infinity exponentially as the critical point is approached, the behavior of $D^{\prime}$ and $D^{\prime \prime}$ are moderate and their magnitude is still small in the range of temperatures lower than $T_{\text {lim }}$. Therefore, $T_{\lim }$ can be considered as the upper boundary of the region where the temperature dependence of the saturated densities is enough small compared to those in the critical region. Since the temperature dependences of the saturated densities are different between substances, the values for $T_{\text {lim }}$ depend on each equation of state.

It is very helpful for making computer programs to know values for $T_{\text {lim }}$ of various equations of state, because for the temperatures lower than $T_{\text {lim }}$ the triple point densities can always be used as the initial guesses. For several published equations of state, $T_{\lim }$ were determined. Table 1 lists $T_{\text {lim }}$ of each equation of state, as well as the triple point and critical point temperatures, $T_{t r}$ and $T_{c}$. The parameter $\gamma$ is normally set to unity, and only for methanol $\gamma$ is 
Table 1 Upper limit of temperature for which the Newton-Raphson iteration using the liquid and vapor densities at the triple point as initial guesses converges successfully

\begin{tabular}{|c|c|c|c|c|}
\hline Substance & Equation of state & $T_{t r}$ & $T_{\lim }$ & $T_{c}$ \\
\hline Carbon dioxide & Span and Wagner ${ }^{(3)}$ & 216.592 & 303 & 304.1282 \\
\hline Water & Wagner and Pruß $\aleph^{(4)}$ & 273.16 & 606 & 647.096 \\
\hline Methane & Setzmann and Wagner ${ }^{(1)}$ & 90.6941 & 189 & 190.564 \\
\hline Ethane & Bücker and Wagner ${ }^{(9)}$ & 90.368 & 285 & 305.322 \\
\hline Isobutane & Bücker and Wagner ${ }^{(10)}$ & 113.73 & 393 & 407.81 \\
\hline Methanol & de Reuck and Craven ${ }^{(11)}$ & 175.61 & 486 & 513.380 \\
\hline Nitrogen & Span et al. ${ }^{(2)}$ & 63.151 & 125 & 126.192 \\
\hline Argon & Tegeler et al. ${ }^{(12)}$ & 83.8058 & 149 & 150.687 \\
\hline Ammonia & Tillner-Roth et al. ${ }^{(13)}$ & 195.50 & 394 & 405.40 \\
\hline $\mathrm{R}-125$ & Lemmon and Jacobsen ${ }^{(14)}$ & 172.52 & 334 & 339.173 \\
\hline \multicolumn{5}{|c|}{ (Pentafluoroethane) } \\
\hline $\begin{array}{l}\text { R-134a } \\
(1,1,1,2 \text {-Tetraflu }\end{array}$ & $\begin{array}{l}\text { Tillner-Roth and Baehr }{ }^{(8)} \\
\text { roethane) }\end{array}$ & 169.85 & 370 & 374.18 \\
\hline \multicolumn{4}{|c|}{$(1,1,1$-Trifluoroethane) } & 345.857 \\
\hline \multicolumn{5}{|c|}{ (Difluoromethane) } \\
\hline $\begin{array}{l}T_{t r}: \text { triple poin } \\
T_{c}: \text { critical poi } \\
T_{\text {lim }}: \text { upper lim }\end{array}$ & $\begin{array}{l}\text { aperature }[\mathrm{K}] \\
\text { mperature }[\mathrm{K}] \\
\text { temperature }[\mathrm{K}]\end{array}$ & & & \\
\hline
\end{tabular}

set to 0.1 . The average temperature difference between $T_{\lim }$ and $T_{c}$ is about $11.6 \mathrm{~K}$. For the temperatures over $T_{\text {lim }}$, it is possible for all equations of state to obtain desirable convergence by using the saturated densities at $T_{\lim }$ as initial guesses.

\section{Conclusion}

For a determination of saturation state using Helmholtz energy equations of state, a useful and reliable method is presented. The commonly used method requires an aid of ancillary equations to estimate accurate initial guesses for the saturation pressure and saturated liquid and vapor densities.

The method presented here is based on the Newton-Raphson algorithm for simultaneous equations. The method can determine the saturation state from the triple point to the critical point, only from the saturated density information at the triple point and at the upper limit of temperature listed in Table 1. No ancillary equation for estimating initial guesses is used in the method. The iterative process is stable, even at the temperature near the critical point. The method can greatly reduce an effort of computer programming.

\section{References}

( 1 ) Setzmann, U. and Wagner, W., Journal of Physical and Chemical Reference Data, Vol.20, (1991), pp.1061-1155.

( 2 ) Span, R., Lemmon, E. W., Jacobsen, R. T., Wagner, W., and Yokozeki, A., Journal of Physical and Chemical Reference Data, Vol.29, (2000), pp.1361-1433.

( 3 ) Span, R. and Wagner, W., Journal of Physical and Chemical Reference Data, Vol.25, (1996), pp.1509-1597.

( 4 ) Wagner, W. and Pruß, A., Journal of Physical and Chemical Reference Data, Vol.31, (2002), pp.387-535.

( 5 ) Lemmon, E. W., Huber, M. L., and McLinden, M. O., NIST Standard Reference Database 23 (REFPROP): Version 8.0, (2007).

( 6 ) Ito, T., Takata, Y., Honda, T., Akasaka, R., Yamaguchi, T., and Momoki, S., PROPATH - A Program Package for Thermophysical Properties of Fluids: Version 13.1, (2008).

( 7 ) Span, R., Multiparameter Equations of State: An Accurate Source of Thermodynamic Property Data, (2000), Springer.

( 8 ) Tillner-Roth, R. and Baehr, H. D., Journal of Physical and Chemical Reference Data, Vol. 23, (1994), pp.657-729. 
( 9 ) Bucker, E. and Wagner, W., Journal of Physical and Chemical Reference Data, Vol.35, (2006), pp.205-266.

(10) Bucker, E. and Wagner, W., Journal of Physical and Chemical Reference Data, Vol.35, (2006), pp.929-1019.

(11) de Reuck, K. M. and Craven, R. J. B., International Thermodynamic Tables of the Fluid State - 12 Methanol, (1993), Blackwell.

(12) Tegeler, Ch., Span, R., and Wagner, W., Journal of Physical and Chemical Reference Data, Vol.28, (1999), pp.779-850.

(13) Tillner-Roth, R., Harms-Watzenberg, F., and Baehr, H. D., DKV-Tagungsbericht, Vol.20, (1993), pp.167-181.

(14) Lemmon, E. W. and Jacobsen, R. T., Journal of Physical and Chemical Reference Data, Vol.34, (2005), pp.69-108.

(15) Lemmon, E. W. and Jacobsen, R. T., Journal of Physical and Chemical Reference Data, Vol.29, (2000), pp.521-552.

(16) Tillner-Roth, R. and Yokozeki, A., Journal of Physical and Chemical Reference Data, Vol.26, (1997), pp.1273-1328. 